PROCEEDINGS OF THE

AMERICAN MATHEMATICAL SOCIETY

Volume 136, Number 11, November 2008, Pages 4003-4010

S 0002-9939(08)09397-0

Article electronically published on June 26, 2008

\title{
ASYMPTOTICALLY HYPERBOLIC METRICS ON A UNIT BALL ADMITTING MULTIPLE HORIZONS
}

\author{
ZHENYANG LI, YUGUANG SHI, AND PENG WU
}

(Communicated by Richard A. Wentworth)

\begin{abstract}
In this paper, we construct an asymptotically hyperbolic metric with scalar curvature -6 on the unit ball $\mathbf{D}^{3}$, which contains multiple horizons.
\end{abstract}

\section{INTRODUCTION}

In general relativity, the initial data set of the Cauchy problem for Einstein equations which is denoted by $\left(M, g_{i j}, p_{i j}\right)$ is of great importance. Here $\left(M, g_{i j}\right)$ is a complete Riemannian 3-manifold and $p_{i j}$ is a symmetric 2-tensor on $M$ satisfying constrain equations (see [7). Among all of the initial data sets, those with asymptotically flat (AF) (see [7]) and asymptotically hyperbolic (AH) (see Definition 2.1) metrics are of most interest so far.

On the other hand, a horizon, which is defined by a surface $\Sigma \subset M$ satisfying $H_{\Sigma}=\operatorname{tr}_{\Sigma}(p)$ (see [2]), is a very interesting geometric object. When $p=0$ (i.e. time symmetric case), the horizon is nothing but a minimal surface. The Schwarzschild and anti-de Sitter-Schwarzschild space are the simplest examples for $\mathrm{AF}$ and $\mathrm{AH}$ manifolds with horizon, respectively. But they both have nontrivial topology. However, for physical and mathematical reasons, people intend to construct topologically trivial manifolds with horizons. In [1, R. Beig and N. Ó Murchadha show that there exists an AF metric which contains a horizon, with a scalar flat on $\mathbb{R}^{3}$. Also, Miao in [6] constructs the same kind of AF manifolds by making use of the Schwarzschild metric and the conformal deformation. These results offer examples of globally regular and AF initial data for the Einstein vacuum equations with minimal surfaces. Combining the method of Miao with that of Chruściel and Delay [3, the author of [4] gives an example of a scalar flat AF metric on $\mathbb{R}^{3}$ admitting multiple horizons.

In recent years, AH manifolds have drawn more and more attention of both mathematicians and physicists. They arise when considering solutions to the Einstein field equations with a negative cosmological constant or when considering "hyperboloidal hypersurfaces" in space-times which are asymptotically flat in isotropic directions. The horizons in AH manifolds are more than minimal surfaces, since

Received by the editors March 29, 2007, and, in revised form, October 11, 2007.

2000 Mathematics Subject Classification. Primary 83C57; Secondary 53C44.

Key words and phrases. Asymptotically hyperbolic metric, horizon, hyperbolic space.

The research of the second author was partially supported by the 973 Program (2006CB805905) and the Fok YingTong Education Foundation.

(C) 2008 American Mathematical Society 4003

Reverts to public domain 28 years from publication 
AH manifolds can be realized as asymptotically null spacelike hypersurfaces in asymptotically flat space-time. Therefore, in an AH manifold, horizons refer not only to boundaries of domains which are minimal surfaces (in the case of considering a negative cosmological constant) but also to boundaries satisfying $H= \pm 2$ (in the case of an asymptotically null spacelike hypersurface in AF spacetimes). Recently, in [8], the authors provide an example of an AH manifold with a constant scalar curvature -6 and horizons (see also Theorem 2.2). Their main idea is to glue the anti-de Sitter-Schwarzchild space with a ball and deform it conformally several times; then they get the desired manifold. Furthermore, they can prove that the mass of their example can be arbitrarily large or small. So, it is natural to consider constructing such an AH manifold admitting multiple horizons. More precisely, in this paper we show that there exists an $\mathrm{AH}$ metric on the unit ball $\mathbf{D}^{3}$ with constant scalar curvature $R=-6$ and multiple horizons (see Theorem 2.4). First, we will construct a metric on a unit 3-ball with multiple horizons using the cut-and-glue method. Secondly, we will conformally deform the metric to an AH metric with constant scalar curvature $R=-6$ by solving a nonlinear PDE; then, as in [8], the existence of horizons follows from the implicit function theorem. We'd like to remark that it seems that our method should work for the construction of an AF manifold with multiple horizons, as has been done in 4 .

The outline of this paper is as follows. In Section 2 we cut the parts containing horizons from some examples of $\mathrm{AH}$ manifolds given by $[8$ and glue them together smoothly. Consequently, we obtain a new AH metric with multiple horizons. In Section 3, we perturb the new metric conformally to an AH metric with constant scalar curvature -6 . Then by keeping the location of horizons far enough from each other, we show that the existence of the multiple horizons is guaranteed by a lemma in 8 .

\section{Construction of an Asymptotically hyperbolic metric ON A UNIT BALL WITH MULTIPLE HORIZONS}

In this section, we will complete the first step of the proof of the main result; namely, we will construct an asymptotically hyperbolic metric on $\mathbf{D}^{3}$ admitting multiple horizons by gluing arguments, but the scalar curvature may not be equal to -6 . First of all, let us recall some basic definitions and facts.

Definition 2.1. A complete non-compact Riemannian manifold $\left(X^{3}, g\right)$ is said to be asymptotically hyperbolic if there is a compact manifold $(\bar{X}, \bar{g})$ with boundary $\partial X$ and a smooth function $t$ on $\bar{X}$ such that the following are true:

(i) $X=\bar{X} \backslash \partial X$.

(ii) $t=0$ on $\partial X$, and $t>0$ on $X$.

(iii) $\bar{g}=t^{2} g$ extends to be $C^{3}$ up to the boundary.

(iv) $|d t|_{\bar{g}}=1$ at $\partial X$.

(v) Each component $\Sigma$ of $\partial X$ is the standard two sphere $\left(\mathbb{S}^{2}, g_{0}\right)$, and there is a collar neighborhood of $\Sigma$ where

$$
g=\sinh ^{-2} t\left(d t^{2}+g_{t}\right)
$$


with

$$
g_{t}=g_{0}+\frac{t^{3}}{3} h+O\left(t^{4}\right)
$$

where $h$ is a $C^{2}$ symmetric two tensor on $\mathbb{S}^{2}$.

It is proved in 9 that for an AH manifold $\left(X^{3}, g\right)$ with scalar curvature $R_{g} \geq-6$, the mass of an end of $X$ corresponding to a boundary component $\Sigma$ of $\partial X$ is welldefined and given by

$$
M=\frac{1}{16 \pi}\left[\left(\int_{\mathbb{S}^{2}} t r_{g_{0}}(h) d v_{g_{0}}\right)^{2}-\left|\int_{\mathbb{S}^{2}} t r_{g_{0}}(h)(x) x d v_{g_{0}}\right|^{2}\right]^{\frac{1}{2}},
$$

where $x$ is the standard coordinates of a point on $\mathbb{S}^{2}$ in $\mathbb{R}^{3}$.

We denote the standard hyperbolic space by $\mathbb{H}^{3}$ and introduce the ball model for $\mathbb{H}^{3}$ which is denoted by $\left(\mathbf{D}^{3}, d s_{\mathbb{H}^{3}}^{2}\right)$. Here, $\mathbf{D}^{\mathbf{3}}$ is the unit ball in $\mathbb{R}^{3}$, and $d s_{\mathbb{H}^{3}}^{2}$ is the standard hyperbolic metric which is defined as follows:

$$
d s_{\mathbb{H}^{3}}^{2}=\frac{4}{\left(1-|x|^{2}\right)^{2}} \sum_{i=1}^{3}\left(d x^{i}\right)^{2},
$$

where $\sum_{i=1}^{3}\left(d x^{i}\right)^{2}$ is the Euclidean metric.

In [8], the authors construct a family of asymptotically hyperbolic metrics on $\mathbf{D}^{3}$ as follows:

Theorem $2.2(8])$. Let $\mathbf{D}^{3}$ be the unit ball in $\mathbb{R}^{3}$. For any $M>0$ and $\delta$, there is a smooth complete metric $g$ on $\mathbf{D}^{3}$ with constant scalar curvature -6 such that the following are true:

(i) $\left(\mathbf{D}^{3}, g\right)$ is asymptotically hyperbolic with mass $M_{g}$ satisfying $\left|M_{g}-M\right|<\delta$.

(ii) There exist surfaces $S_{1}, S_{2}$ and $S_{3}$ which are topological spheres with constant mean curvature -2, 0,2 respectively such that $S_{1}$ is in the interior of $S_{2}$ and $S_{2}$ is in the interior of $S_{3}$.

(iii) Outside a compact set $U$, the metric $g$ is conformal to the standard hyperbolic metric of $\mathbf{D}^{3}$, and $S_{1}, S_{2}$ and $S_{3}$ are contained in $\mathbf{D}^{3} \backslash U$.

Let us fix some notation for our paper. We will denote the origin by $o$. Let $x \in \mathbf{D}^{3}$ and $B_{x}(\rho)$ be the geodesic ball centered at $x$ with radius $\rho$ under the standard hyperbolic metric on $\mathbf{D}^{3}$. The hyperbolic distance starting from $x$ to $y$ will be denoted by $\rho_{x}(y)$ (simply by $\rho(y)$ if $x$ is the origin). Without loss of generality, we may reformulate Theorem 2.2 in the following way:

Theorem 2.3. Let $S_{1}, S_{2}, S_{3}$ be the surfaces and $g$ be the $A H$ metric which are given by Theorem 2.2. Then there exists a geodesic ball $B_{o}(\delta)$ under the hyperbolic metric such that $S_{1}, S_{2}, S_{3}$ are contained in $B_{o}(\delta) \backslash B_{o}\left(\frac{\delta}{2}\right)$ and $g$ is conformal to the standard hyperbolic metric of $\mathbf{D}^{3}$ on $\mathbf{D}^{3} \backslash B_{o}\left(\frac{\delta}{4}\right)$.

Using the gluing method and conformal deformation again, we are able to prove our main result:

Theorem 2.4. Let $\mathbf{D}^{3}$ be the unit open ball in $\mathbb{R}^{3}$. For any $K>0$, there is an $A H$ metric $g$ on $\mathbf{D}^{3}$ with constant scalar curvature -6 such that there are $\left\{x_{k}\right\}_{k=1}^{K} \subset \mathbf{D}^{3}$ and surfaces $S_{1}^{i}, S_{2}^{i}$ and $S_{3}^{i}, 1 \leq i \leq K$, which are topological spheres with constant mean curvature $-2,0,2$ respectively and are contained in $B_{x_{i}}(\delta)$ and which do not 
intersect each other. Moreover, $S_{1}^{i}$ is in the interior of $S_{2}^{i}$, and $S_{2}^{i}$ is in the interior of $S_{3}^{i}$; and outside a compact set, the metric $g$ is conformal to the standard hyperbolic metric of $\mathbf{D}^{3}$.

We consider only the case for $K=2$, since the other cases are essentially the same. By gluing arguments, we will show

Proposition 2.5. There is a smooth $A H$ metric $\widetilde{g}$ on $\mathbf{D}^{3}$ with the following properties:

(1) $B_{o}\left(\delta_{2}\right) \backslash B_{o}\left(\frac{\delta_{2}}{2}\right)$ and $B_{p}\left(\delta_{1}\right) \backslash B_{p}\left(\frac{\delta_{1}}{2}\right)$ each contain the surfaces with mean curvature $2,0,-2$. Here, $o, p$ are two points in $\mathbf{D}^{3}$, with hyperbolic distance being $2 \tau \triangleq \rho(p)>10\left(\delta_{1}+\delta_{2}\right)$, so that $B_{o}\left(\delta_{2}\right)$ and $B_{p}\left(\delta_{1}\right)$ do not intersect each other. Without loss of generality, we may assume $\tau \geq 100$.

(2) The scalar curvature $R_{\widetilde{g}}$ of $\widetilde{g}$ satisfies that

$$
R_{\widetilde{g}}(x)=-6
$$

for $x \in \mathbf{D}^{3} \backslash\left(B_{p}(\tau+2) \backslash B_{p}(\tau+1)\right)$ and

$$
\left|R_{\widetilde{g}}(x)+6\right| \leq C e^{-3 \tau}
$$

for $x \in B_{p}(\tau+2) \backslash B_{p}(\tau+1)$. Here, $C$ is a positive constant which is independent of $\tau$.

(3) $\widetilde{g}$ is conformal to the hyperbolic metric outside $B_{o}\left(\frac{\delta_{2}}{4}\right) \cup B_{p}\left(\frac{\delta_{1}}{4}\right)$.

Proof. Choose two AH metrics $g_{1}, g_{2}$ as given by Theorem 2.2 (not necessarily having the same mass). Let $B_{o}\left(\delta_{1}\right)$ and $B_{o}\left(\delta_{2}\right)$ be the sets described in Theorem 2.3 for $\left(\mathbf{D}^{3}, g_{1}\right)$ and $\left(\mathbf{D}^{3}, g_{2}\right)$ respectively such that for $i=1,2$,

$$
g_{i}(y)=\phi_{i}^{4}(y) d s_{\mathbb{H}^{3}}^{2},
$$

where $y \in \mathbf{D}^{3} \backslash B_{o}\left(\frac{\delta_{i}}{4}\right)$. Also, by Theorem 4.1 in [8], $\phi_{i}$ satisfies

$$
\left\|\phi_{i}(y)-1\right\|_{\mathbf{C}^{3}} \leq C e^{-3 \rho(y)}
$$

for $y \in \mathbf{D}^{3} \backslash B_{o}\left(\frac{\delta_{i}}{4}\right)$, and $C$ is a positive constant that is independent of $y$.

Now, we will glue $g_{1}$ and $g_{2}$ together as follows.

Let us introduce the upper halfspace model $\mathbb{R}_{+}^{3}$ for $\mathbb{H}^{3}$ and label the point $x \in \mathbb{H}^{3}$ by $(\mathbf{x}, y)$ with $\mathbf{x} \in \mathbb{R}^{2}$ and $y \in \mathbb{R}_{+}$. Under this coordinate system, the standard metric for hyperbolic space can be expressed as

$$
d s_{\mathbb{H}^{3}}^{2}=\frac{\left(d x^{1}\right)^{2}+\left(d x^{2}\right)^{2}+d y^{2}}{y^{2}}
$$

here, $\mathbf{x}=\left(x^{1}, x^{2}\right) \in \mathbb{R}^{2}$. Suppose $o=(\mathbf{0}, 1)$ and $p=\left(\mathbf{x}_{p}, y_{p}\right)$. Then there is a hyperbolic translation $F$ which maps $o$ to $p$ with $\rho(p) \triangleq 2 \tau>10\left(\delta_{1}+\delta_{2}\right)$,

$$
\begin{aligned}
F: B_{o}(\tau+3) & \longrightarrow B_{p}(\tau+3), \\
(\mathbf{x}, y) & \longmapsto F(\mathbf{x}, y)=\left(\mathbf{x}_{p}+y_{p} \mathbf{x}, y_{p} y\right) .
\end{aligned}
$$

Then it is easy to see that $F$ induces a natural isometry between the standard hyperbolic metric in $B_{p}(\tau+3)$ and that in $B_{o}(\tau+3)$ :

$$
\left(B_{p}(\tau+3),\left.\left(F^{-1}\right)^{*} d s_{\mathbb{H}^{3}}^{2}\right|_{B_{0}(\tau+3)}\right) \cong\left(B_{p}(\tau+3),\left.d s_{\mathbb{H}^{3}}^{2}\right|_{B_{p}(\tau+3)}\right) .
$$

Indeed, $\left(F^{-1}\right)^{*}\left(\left.d s_{\mathbb{H}^{3}}^{2}\right|_{B_{0}(\tau+3)}\right)=\left.d s_{\mathbb{H}^{3}}^{2}\right|_{B_{p}(\tau+3)}$ in the sense that the metrics on both sides have the same components under the standard upper halfspace coordinates. 
Therefore, we can pull $g_{1}$ on $B_{o}(\tau+3)$ to $B_{p}(\tau+3)$ by the diffeomorphism $F$, which gives an isometry:

$$
\left(B_{p}(\tau+3),\left(F^{-1}\right)^{*} g_{1}\right) \cong\left(B_{o}(\tau+3), g_{1}\right)
$$

Because of $(2.2)$, we can identify $B_{o}(\tau+3)$ and $B_{p}(\tau+3)$ both equipped with hyperbolic metric via $F$, and

$$
\left(F^{-1}\right)^{*} g_{1}=\left(\phi_{1} \circ F^{-1}\right)^{4}\left(F^{-1}\right)^{*}\left(\left.d s_{\mathbb{H}^{3}}^{2}\right|_{B_{0}(\tau+3)}\right)=\left.\left(\phi_{1} \circ F^{-1}\right)^{4} d s_{\mathbb{H}^{3}}^{2}\right|_{B_{p}(\tau+3)}
$$

for $x \in B_{p}(\tau+3) \backslash B_{p}\left(\frac{\delta_{1}}{4}\right)$. Also, the inequality (2.1) for $\phi_{1}$ can be described as

$$
\left\|\phi_{1} \circ F^{-1}(y)-1\right\|_{\mathbf{C}^{3}} \leq C e^{-3 \rho_{p}(y)}
$$

for $y \in B_{p}(\tau+3) \backslash B_{p}\left(\frac{\delta_{1}}{4}\right)$. For simplicity, $\phi_{1} \circ F^{-1}(y)$ will still be denoted by $\phi_{1}(y)$ in the sequel.

Let $\eta$ be a smooth cut-off function such that $0 \leq \eta \leq 1$ and

$$
\eta(x)= \begin{cases}1 & x \in B_{p}(\tau+1) \\ 0 & x \in \mathbf{D}^{3} \backslash B_{p}(\tau+2) .\end{cases}
$$

Hence $\|\eta\|_{C^{2}}$ is uniformly bounded. Next, we define a new metric $\widetilde{g}$ on $\mathbf{D}^{3}$, which is given by

$$
\widetilde{g}(x)= \begin{cases}\left(F^{-1}\right)^{*} g_{1}, & x \in B_{p}(\tau+1) ; \\ \left.\left(\eta \phi_{1}+(1-\eta) \phi_{2}\right)^{4} d s_{\mathbb{H}^{3}}^{2}\right|_{B_{p}(\tau+2) \backslash B_{p}(\tau+1),}, & x \in B_{p}(\tau+2) \backslash B_{p}(\tau+1) ; \\ g_{2}, & x \in \mathbf{D}^{3} \backslash B_{p}(\tau+2) .\end{cases}
$$

By its definition, we see $\widetilde{g}$ satisfies (1) in Proposition 2.5.

Again by the definition of $\widetilde{g}$ and (2.1), we can calculate that the scalar curvature $R_{\widetilde{g}}$ of $\widetilde{g}$ satisfies that

$$
R_{\widetilde{g}}(x)=-6 \quad \text { for } \quad x \in \mathbf{D}^{3} \backslash\left(B_{p}(\tau+2) \backslash B_{p}(\tau+1)\right)
$$

and for $x \in B_{p}(\tau+2) \backslash B_{p}(\tau+1)$,

$$
\left|R_{\widetilde{g}}(x)+6\right| \leq C e^{-3 \tau}
$$

Thus, we verified (2) in Proposition 2.5, and by Theorem 2.3, we see that (3) in Proposition 2.5 is also true; therefore, we finish to prove the proposition.

Remark 2.6. One can see from the construction of $\widetilde{g}$ that it depends on $\tau$. To emphasize this, we will denote $\widetilde{g}$ by $\widetilde{g}_{\tau}$ in the next section.

\section{Proof of the main Result by Conformal Deformation}

In this section, we will prove our main result of Theorem 2.4. Namely, we perturb $\widetilde{g}_{\tau}$ constructed in the last section by conformal deformation and show that the resulting metric is an $\mathrm{AH}$ metric with scalar curvature equal to -6 and containing multiple horizons. For this purpose, we need

Lemma 3.1. Let $\widetilde{g}_{\tau}$ be constructed as in Proposition 2.5. Then there is $u_{\tau}>0$ such that $g_{\tau}=u_{\tau}^{4} \widetilde{g}_{\tau}$ is an AH metric with scalar curvature $R=-6$, and

$$
\lim _{\tau \rightarrow \infty} \sup _{\mathbf{D}^{3}}\left|u_{\tau}-1\right|=0 \text {. }
$$

Moreover, outside a compact set, the metric $g_{\tau}$ is conformal to the standard hyperbolic metric on $\mathbf{D}^{3}$. 
Proof. It is sufficient to solve the following equation:

$$
\left\{\begin{array}{l}
\triangle_{\tilde{g}_{\tau}} u-\frac{3}{4} u^{5}-\frac{1}{8} R_{\widetilde{g}_{\tau}} u=0, \\
\lim _{\rho(x) \rightarrow+\infty} u(x)=1
\end{array}\right.
$$

To do this, we will use exhausting domain arguments. Let us choose a sequence $\left\{\rho_{k}\right\}_{k=1}^{\infty}$ with $\rho_{1} \geq 3 \tau$ such that $\rho_{k}<\rho_{k+1}$ for $k \in \mathbf{N}$ and $\rho_{k} \rightarrow+\infty$ as $k \rightarrow+\infty$. Consider the following Dirichlet problem:

$$
\left\{\begin{array}{l}
\triangle_{\widetilde{g}_{\tau}} u_{i}-\frac{3}{4} u_{i}^{5}-\frac{1}{8} R_{\widetilde{g}_{\tau}} u_{i}=0, \quad \text { on } \quad B_{o}\left(\rho_{i}\right), \\
\left.u_{i}\right|_{\partial B_{o}\left(\rho_{i}\right)}=\phi_{2}^{-1} .
\end{array}\right.
$$

By the standard variational method, we see (3.2) has a smooth nonnegative solution. Also, by the maximal principle, $u_{i}$ must be positive. By assumption for $\phi_{2}$, we know that $1-C e^{-3 \rho_{i}} \leq\left. u_{i}\right|_{\partial B_{o}\left(\rho_{i}\right)} \leq 1+C e^{-3 \rho_{i}}$. We claim that

$$
\sup _{B_{o}\left(\rho_{i}\right)}\left|u_{i}-1\right| \leq C e^{-3 \tau}
$$

here, $C$ is independent of $i$ and $\tau$. Let us prove that the lower bounded estimate is true. Indeed, suppose $u$ attains its minimum at $x_{0}$. If $x_{0} \in \partial B_{o}\left(\rho_{i}\right)$, then the claim of the lower bound follows; otherwise, at an interior point $x_{0}$, one has

$$
0 \leq u_{i}^{-1} \triangle_{\widetilde{g}_{\tau}} u_{i}=\frac{3}{4} u_{i}^{4}+\frac{1}{8} R_{\widetilde{g}_{\tau}} .
$$

By (2) of Proposition 2.5, we get the claim for the lower bound. By similar arguments, we will get the upper bounded estimate. Thus the claim is true. Then, by exhausting domain, we get the solution $u_{\tau}$ of $(3.1)$, and

$$
\sup _{\mathbf{D}^{3}}\left|u_{\tau}-1\right| \leq C e^{-3 \tau}
$$

here, $C$ is a constant which is independent of $\tau$.

Next, we will construct a barrier of the equation at infinity of the manifold, and by using this we show that $u$ approaches 1 at a desired rate.

Let $v_{i}=u_{i} \phi_{2}$. Since $g_{2}$ is conformal to the standard hyperbolic metric outside $B_{o}(3 \tau)$, we have

$$
\left\{\begin{array}{l}
\mathbf{L}\left(v_{i}\right):=\triangle_{\mathbb{H}^{3}} v_{i}-\frac{3}{4} v_{i}\left(v_{i}^{4}-1\right)=0, \quad \text { for } \quad x \in B_{o}\left(\rho_{i}\right) \backslash B_{o}(\bar{\rho}), \\
\left.v_{i}\right|_{\partial B_{o}\left(\rho_{i}\right)}=1
\end{array}\right.
$$

for some $\bar{\rho} \geq 3 \tau$, where $\triangle_{\mathbb{H}^{3}}$ is the Laplacian operator for hyperbolic metric. Set $f_{-}(x)=1-\lambda e^{-3 \rho(x)}$, for $\lambda \geq 0$. Hence,

$$
\begin{aligned}
\mathbf{L}\left(f_{-}\right)= & -9 \lambda e^{-3 \rho(x)}+6 \lambda e^{-3 \rho(x)} \operatorname{coth} \rho \\
& -\frac{3}{4}\left(1-\lambda e^{-3 \rho(x)}\right)\left[\left(1-\lambda e^{-3 \rho(x)}\right)^{4}-1\right] \\
= & 6 \lambda(\operatorname{coth} \rho-1) e^{-3 \rho(x)}+O\left(e^{-6 \rho(x)}\right) .
\end{aligned}
$$

Since $(\operatorname{coth} \rho-1) e^{-3 \rho(x)}>2 e^{-5 \rho(x)}$, for sufficiently large $\bar{\rho}$ we have $\mathbf{L}\left(f_{-}\right)>0$ in $B_{o}\left(\rho_{i}\right) \backslash B_{o}(\bar{\rho})$. So let us choose $\lambda=e^{3 \bar{\rho}}$; then $\mathbf{L}\left(f_{-}\right)>0$ whenever $\rho \geq \bar{\rho}, v_{i}=1 \geq$ $f_{-}$at $\partial B_{o}\left(\rho_{i}\right)$, and $v_{i} \geq f_{-}=0$ at $\partial B_{o}(\bar{\rho})$. Due to (2.1) and (3.3), by choosing $\tau$ and $\bar{\rho}$ sufficiently large, we may assume $v_{i} \geq 2^{-\frac{1}{2}}>5^{-\frac{1}{4}}$ on $B_{o}\left(\rho_{i}\right) \backslash B_{o}(\bar{\rho})$. Now, we claim that $v_{i} \geq f_{-}$on $B_{o}\left(\rho_{i}\right) \backslash B_{o}(\bar{\rho})$. For any point of the boundary, the claim 
is obviously true. Suppose the claim fails. Then for any $p \in B_{o}\left(\rho_{i}\right) \backslash B_{o}(\bar{\rho})$ with $\left(v_{i}-f_{-}\right)(p)=\inf _{B_{o}\left(\rho_{i}\right) \backslash B_{o},(\bar{\rho})}\left(v_{i}-f_{-}\right)<0$ is an interior point at which we have

$$
5^{-\frac{1}{4}}<2^{-\frac{1}{2}} \leq v_{i}(p)<f_{-}(p) .
$$

Thus, at $p$, we have

$$
0 \leq \triangle_{\mathbb{H}^{3}}\left(v_{i}-f_{-}\right) \leq \frac{3}{4} v_{i}\left(v_{i}^{4}-1\right)-\frac{3}{4} f_{-}\left(f_{-}^{4}-1\right)<0,
$$

which is a contradiction. Therefore, we obtain

$$
v_{i}-1 \geq-\lambda e^{-3 \rho} \quad \text { in } \quad B_{o}\left(\rho_{i}\right) \backslash B_{o}(\bar{\rho}) .
$$

On the other hand, by noting the super solution $f_{+}(x)=1+\lambda e^{-3 \rho(x)}$ and using similar arguments, we can prove that $v_{i}-1 \leq \lambda e^{-3 \rho}$ in $B_{o}\left(\rho_{i}\right) \backslash B_{o}(\bar{\rho})$. Hence, we have

$$
\left|v_{i}-1\right| \leq \lambda e^{-3 \rho(x)} \quad \text { in } \quad B_{o}\left(\rho_{i}\right) \backslash B_{o}(\bar{\rho}) .
$$

Consequently, $u_{\tau}$ satisfies that

$$
\left|u_{\tau}-1\right| \leq \lambda e^{-3 \rho} \quad \text { in } \quad \mathbf{D}^{3} \backslash B_{o}(\bar{\rho}),
$$

and also by $(3.3)$

$$
\sup _{\mathbf{D}^{3}}\left|u_{\tau}-1\right| \leq C e^{-3 \tau}
$$

as mentioned above here $C$ is a constant that is independent of $\tau$.

Now applying Lemma 4.2 in 8, we conclude from (3.4) that the manifold $\left(\mathbf{D}^{\mathbf{3}}, g_{\tau}\right)$ with $g_{\tau}=u_{\tau}^{4} \widetilde{g}_{\tau}$ is an AH manifold. Also, it follows from Proposition 2.5 that $g_{\tau}$ is conformal to the hyperbolic metric outside some compact subset of $\mathbf{D}^{3}$. Thus, we finish the proof of the lemma.

Next, we have the following:

Lemma 3.2. Let $g_{\tau}$ be as in Lemma 3.1, which depends on $\tau$. Then for sufficiently large $\tau,\left(\mathbf{D}^{3}, g_{\tau}\right)$ contains surfaces $S_{1}^{i}, S_{2}^{i}$ and $S_{3}^{i}, 1 \leq i \leq 2$, which are topological spheres with constant mean curvature $-2,0,2$ and are contained in $B_{o}(\delta)$ and $B_{p}(\delta)$ respectively which do not intersect each other. Moreover, $S_{1}^{i}$ is in the interior of $S_{2}^{i}$, and $S_{2}^{i}$ is in the interior of $S_{3}^{i}$.

Proof. Let us show that horizons are in $\left(B_{o}(2 \delta), g_{\tau}\right)$ when $\tau$ is large enough. In fact, by Lemma 4.4 in $[8$, it is sufficient to show that

$$
\left\|u_{\tau}-1\right\|_{C^{2, \alpha}\left(B_{o}(2 \delta) \backslash B_{o}\left(\frac{\delta}{4}\right)\right)} \leq \epsilon .
$$

Here $\epsilon$ and $u_{\tau}$ are given in Lemma 4.4 in 8 and Lemma 3.1 respectively. Note in $\left(B_{o}(2 \delta), \widetilde{g}_{\tau}\right)$ that sectional curvature is bounded and the injective radius has a uniform positive lower bound. Then $B_{o}\left(\frac{3}{2} \delta\right)$ can be covered by a finite number of harmonic coordinates which have uniform size (for the existence of harmonic coordinates and estimates of their size, see (5). The number and size of these harmonic coordinates are independent of $\tau$. For any $x \in B_{o}\left(\frac{3}{2} \delta\right)$, without loss of generality, we may assume $B_{x}(1)$ has already been covered by such harmonic coordinates; then the components of the metric $g_{\tau}$ under the harmonic coordinates satisfy

$$
\left\|\left(\widetilde{g}_{\tau}\right)_{i j}\right\|_{C^{1, \alpha}} \leq C
$$


Here $C$ is independent of $\tau$ and $x$. Now in $B_{x}(1)$, we have the equation

$$
\triangle_{\widetilde{g}_{\tau}} u_{\tau}=\frac{3}{4} u_{\tau}\left(u_{\tau}^{4}-1\right),
$$

by (3.1). Then combining (3.5) with the standard estimate of PDE, we get

$$
\left\|u_{\tau}-1\right\|_{C^{2, \alpha}\left(B_{x}(1)\right)} \leq C e^{-3 \tau},
$$

where $C$ is independent of $\tau$. Since $x$ is arbitrary in $B_{o}(2 \delta)$ and $\tau$ can be arbitrarily large, we get (3.6), which implies there are horizons in $B_{o}(2 \delta)$. By the same arguments, we can show there are also horizons in $B_{p}(2 \delta)$. Thus we complete the proof of the lemma.

Combining the above lemmas, we get the proof of Theorem 2.4, which proves our main result.

\section{REFERENCES}

1. Beig, R., and Ó Murchadha, N.: Trapped surfaces due to concentration of gravitational radiation, Phys. Rev. Lett. 66 (1991), no. 19, 2421-2424. MR.1104859 (92a:83005)

2. Bray, H.L., and Chruściel, P.T.: The Penrose Inequality, The Einstein Equations and the Large Scale Behavior of Gravitational Fields, pp. 39-70. Birkhäuser, Basel (2004). MR2098913 (2005m:83014)

3. Chruściel, P.T., and Delay, E.: Existence of non-trivial, vacuum, asymptotically simple spacetimes, Classical Quantum Gravity 19 (2002), no. 9, L71-79; Erratum: Classical Quantum Gravity 19 (2002), no. 12, 3389. MR1902228(2003e:83024a)

4. Corvino, Justin: A note on asymptotically flat metrics on $\mathbb{R}^{3}$ which are scalar-flat and admit minimal spheres, Proc. Amer. Math. Soc. 133 (2005), no. 12, 3369-3678. MR 2163606 (2007a:53077)

5. Jost, J., and Karcher, H.: Geometrische Methoden zur Gewinnung von a-priori-Schranken für harmonische Abbildungen, Manuscripta Math. 40 (1982), no. 1, 27-77. MR679120|(84e:58023)

6. Miao, P.: Asymptotically flat and scalar flat metrics on $\mathbb{R}^{3}$ admitting a horizon, Proc. Amer. Math. Soc. 132 (2004), no. 1, 217-222. MR2021265 (2004m:53065)

7. Schoen, R., and Yau, S.T.: Proof of the positive mass theorem. I, Commun. Math. Phys. 65 (1979), 45-76. MR.526976 (80j:83024)

8. Shi, Yuguang, and Tam, Luen-Fai: On the construction of asymptotically hyperbolic manifolds with horizons, Manuscripta Math. 122 (2007), no. 1, 97-117. MR2287702 (2007m:53036)

9. Wang, X., Mass for asymptotically hyperbolic manifolds, J. Differential Geom. 57 (2001), 273-299. MR1879228 (2003c:53044)

Key Laboratory of Pure and Applied Mathematics, School of Mathematics Science, Peking University, Beijing, 100871, People's Republic of China

E-mail address: lzymath@163.com

Current address: School of Sciences, Hangzhou Dianzi University, Xiasha Hangzhou, Zhejiang, 310018, People's Republic of China

Key Laboratory of Pure and Applied mathematics, School of Mathematics Science, Peking University, Beijing, 100871, People's Republic of China

E-mail address: ygshi@math.pku.edu.cn

Key Laboratory of Pure and Applied Mathematics, School of Mathematics Science, Peking University, Beijing, 100871, People's Republic of China

E-mail address: wupenguin@gmail.com

Current address: Department of Mathematics, University of California, Santa Barbara, Santa Barbara, California 93106 